\title{
Medical-surgical collaboration in the practice of gastroenterology
}

\author{
W. SIRCUS \\ M.D., Ph.D. F.R.C.P.(Lond), F.R.C.P.(Edin.) \\ Gastro-intestinal Unit, University of Edinburgh, Western General Hospital, Edinburgh EH4 $2 X U$
}

Oath of Hippocrates 460-355 BC: 'I will not use the knife, not even, verily on sufferers from stone, but I will give place to such as are craftsmen therein.'

\section{Introduction}

Clearly physicians have known their place for millenia. Nevertheless, the relatively modern convention of separating (in hospital) patients into surgical and non-surgical reflected as much proprietorial restrictions and the historical origin of the surgeons as barbers as it reflected any consideration of advantage to the patient. Furthermore, the different approach to disease between the practitioners of the two disciplines reflects to a remarkable degree the differences in personality which appear to direct the individual doctor to choice of specialty. The expression that 'we are all physicians but some of us operate also' smacks more of piety than conviction.

It is therefore of interest that in 1937 at the founding meeting of the Gastroenterological Club by Sir Arthur Hurst it was resolved that as the club numbered one radiologist, one morbid anatomist and one biochemist of eminence, a surgeon occupying a similar standing should be elected' and it was agreed to invite Sir David Wilkie. There were 36 physicians including Francis Avery Jones.

With respect to the subject of this paper, it rapidly became apparent to the writer that only a limited purpose could be served by an examination of collaborative exercises as reported in the journals and, while the essence of the subject demanded evaluation of the unconventional against the conventional, no meaning could attach to comparing the unlike. For these reasons the second half of this paper is concerned with the Edinburgh unit which, perhaps still uniquely, reflects the principle of total collaboration between the disciplines to the point of loss of personal sovereignty.

In the UK in the period 1925-1945 formalized collaboration between physicians and surgeons for the investigation and treatment of disease was encountered in clinics for respiratory disorders, tuberculosis in particular. Joint discussion of individual cases with decisions on management was the practice in the London Chest Hospital, in the Thoracic Unit at Broadgreen Hospital in Liverpool and elsewhere. During the latter half of that period, in the Central Middlesex Hospital, Avery Jones had come to the opinion that improvement in the results of managing acute disorders of the alimentary tract was also dependent on close collaboration between physicians and surgeons. He therefore developed a special relationship with his surgical colleagues, in particular J. W. P. Gummer, for the investigation and care of gastrointestinal problems which could require surgery, in particular bleeding from the upper alimentary tract. Cases could be submitted for joint consideration and subsequent collaborative management and if cases admitted to the medical ward in the care of the physicians required surgery they remained in that medical charge after the operation.

The Central Middlesex department continues the arrangement under George Misiewicz and his colleagues. Close collaboration between physicians and surgeons for the management of gastrointestinal disease was set up by Charles Clark, Professor of Surgery at University College Hospital in the early sixties, at St Mark's Hospital in 1965 on the appointment of a physician, John Lennard-Jones, at the Frenchay Hospital in Bristol by Roger Celestin, on Teeside by Alex Dellipiani and in Northwick Park Hospital by Jonathan Levi and Alan Cox. Last year Michel Cremer set up a combined unit in Brussels. Each of these arrangements involves one consultant physician together with one or two consultant surgeons. Thus the concept of close collaboration can no longer be regarded as experimental. There are probably other units around the world with similar arrangements of which the writer is unaware.

The evaluation of the results of combined care and the comparison of them with those from centres with conventional arrangements raises a number of problems. The simple expression of results in terms of morbidity and mortality of disease is unsatisfactory as units with special arrangements, facilities and 
TABLE 1. The percentage mortality from gastroduodenal haemorrhage according to different policies of management at St James' Hospital, London, 1941-49 (after Tanner, 1950)

\begin{tabular}{lcccc}
\hline Periods & $1941-43$ & $1943-44$ & $1944-47$ & $1948-49$ \\
\hline Policy & Avoid operation & Early partial & Operate early, & Operate immediately \\
if possible & 193 & gastrectomy & simplest procedure & if known to have ulcer \\
Number of cases & $10 \%$ & 60 & 312 & 183 \\
Overall mortality & $20 \%$ & $11 \%$ & $7 \%$ \\
\hline
\end{tabular}

expertise attract more severe and complicated problems, often as secondary, even tertiary, referrals. Vertical movement of the statistics of morbidity and mortality is also barely interpretable as with the passage of time diseases change in severity and character and advances appear in management. For example, as an expression of these variables, in idiopathic colitis from 1950-1967 the Edinburgh Gastro-intestinal Unit treated 55 cases of toxic dilatation of the colon of whom 25 died. From 1968-1982 13 cases were managed with no deaths. There is no evidence that these differences reflected the extended experience of a collaborative approach to the management, though they may be associated with better and more effective antibiotic treatment and improved methods of maintaining nutrition and the normal metabolic state. Thus interpreting change of results in treating a particular disease has to account for changes in the natural history of that disease, especially in its severity, as well as in the modes of management.

In the last decade the developments in diagnostic and therapeutic endoscopy have blurred the division between the disciplines. Both physicians and surgeons (and others) now deal with oesophageal strictures, benign and malignant, dilating one and intubating the other, and with bleeding oesophageal varices. They both snare polyps and coagulate with light or heat bleeding vessels exposed by peptic ulceration. Both may now intubate biliary and pancreatic tracts and remove calculi from them; both resect polyps and tumours in the colon and both remove foreign bodies from either end of the alimentary canal.

\section{Upper gastrointestinal bleeding}

The two conditions of alimentary disease which might be regarded as particularly likely to gain advantage from medical and surgical collaboration are inflammatory bowel disease and bleeding peptic ulcers.

Because of the evaluation difficulties mentioned above it was not possible to make a comparison between the results of treating inflammatory bowel disease in conventional circumstances and those in which the collaboration of physicians and surgeons was a dominant feature. On the other hand a number of reports of the management of bleeding from the upper alimentary tract have appeared in recent years in which collaboration was a specific and notable o feature. These reports, therefore, have been used to 8 examine the contribution from collaboration in the 3 context of disease management. This is also appropriate as the earliest collaborative exercise mounted by Avery Jones at the Central Middlesex Hospital $\odot$ was concerned with just this subject of alimentary bleeding.

Seven major reports published between 1950 and 1983 have been examined, five of which originated from collaborative arrangements.

The first, that from Norman Tanner (1950) illustrates the difficulty in evaluating results between series: in this one four changes of management are compared over a period of 9 years (Table 1).

In 1956 Avery Jones in his memorial lecture to the American Gastroenterological Association reporte⿳亠丷厂 $₫$ the results of treating alimentary haemorrhage at the Central Middlesex Hospital over two eras: the first 1941-1946 when a conservative policy prevailed and $\bar{\partial}$ 1947-1954 when the policy was changed, to proceed to surgery if the patient was over 40 years of age and $\mathbb{\square}$ experienced continued or recurrent bleeding after $\overrightarrow{\vec{P}}$ admission (Table 2). It is clear that the change of policy had little effect upon mortality, the outstanding influence on that being the age of the patient.

TABLE 2. The percentage overall mortality of bleeding peptic ulcer in the Central Middlesex Hospital through two eras and with changes in management policy (see text, after Avery Jones, 1956)

\begin{tabular}{lccc}
\hline Site of ulcer & Age in years & $\begin{array}{c}1941-46 \\
\text { Percentage }\end{array}$ & $\begin{array}{c}1951-54 \\
\text { Percentage }\end{array}$ \\
\hline Gastric & $\left\{\begin{array}{ccc}\text { under } 60 \\
\text { over } 60\end{array}\right.$ & 14.3 & 5.6 \\
Duodenal & $\left\{\begin{array}{c}\text { under 60 } \\
\text { over 60 }\end{array}\right.$ & $2 \cdot 1$ & 21.2 \\
\hline
\end{tabular}

Almost two decades later the mortality in North $O$ east Scotland from gastrointestinal haemorrhage was $\bullet$ reported by an informal but collaborating group of surgeons and a physician (Jones et al., 1973). The? survey was prospective and 817 cases were admitted 
TABLE 3. Mortality from bleeding duodenal ulcer in different eras before and after the establishment in 1972 of a special management unit (see Hunt et al., 1979)

\begin{tabular}{lcccccc}
\hline Period & $1951-60$ & $1961-70$ & $1972-74$ & $1974-76$ & $1976-78$ & Total \\
\hline Number of cases & & & 88 & 101 & 93 & 282 \\
Overall deaths & - & - & 11 & 9 & 3 & \\
Operative deaths & - & - & 5 & 4 & 1 & \\
Surgical mortality & $24 \%$ & $17 \%$ & $13 \%$ & $8 \%$ & $3 \%$ & \\
\hline
\end{tabular}

between 1967-1968. The mortality for bleeding duodenal ulcer was $7 \cdot 1 \%$ and for gastric ulcer $16.9 \%$. If bleeding recurred after admission the mortality rose to $28.8 \%$. The mortality for those with ulcers operated upon was $24 \%$. The collaboration had made little impact upon the statistics.

Three teams of physicians and surgeons with agreed protocols for management of bleeding peptic ulcers, including admission to a centralized unit set aside for the purpose, have reported their results.

The first, from Melbourne (Table 3) included in the protocol endoscopy within $12 \mathrm{hr}$ and automatic surgery for all duodenal ulcer patients over 50 years who were shocked or needed more than five pints of blood (Hunt et al., 1979). Even so 4 years passed before mortality was reduced below the level achieved by units without collaboration. Even in the most favourable period the mortality is similar to that reported from the Central Middlesex Hospital for duodenal ulcer patients under 60 years in 1951-1954.

The second management team, in Glasgow, brought the mortality in bleeding duodenal ulcer down to $8.1 \%$ but 'at the expense' of a high operative mortality for poor risk subjects (Birnie et al., 1981).

Another specially designated team, in Birmingham (Morris et al., 1983), concluded from their experience that no benefit had accrued from the centralized unit staffed by physicians and surgeons (Table 4).

TABLE 4. Mortality from gastroduodenal haemorrhage overall and where due to peptic ulcer in particular, in three eras (after Morris et al., 1983)

\begin{tabular}{lccc}
\hline & $1970-73$ & 1979 & $1980-82$ \\
\hline Number of cases & - & 113 & 387 \\
Overall mortality $(\%)$ & $9 \cdot 7$ & $12 \cdot 4$ & $9 \cdot 8$ \\
Mortality in peptic ulcer $(\%)$ & $6 \cdot 1$ & 6.9 & 4.6 \\
\hline
\end{tabular}

A comparison of a conservative approach to bleeding ulcer versus an active surgical one was made over two periods-1975-1977 and 1978-1980 in Nottingham hospitals (Vellacott et al., 1982). The advent of $\mathrm{H}_{2}$ receptor antagonist drugs appears to have had no influence on the outcome of the second period. No differences were found between the two periods (Table 5).

It has been claimed that without collaborating disciplines the mortality for bleeding duodenal ulcer can be zero if the diagnosis is known before operating and surgery is immediate (Himal et al., 1974) but presumably a proportion of such patients would have responded to conservative measures alone.

These reports demonstrate that collaboration between physicians and surgeons does not automatically confer advantages in management of alimentary disorders. Such factors as arteriosclerosis and obstructive airways disease represent a hazard in patients over 50 years for which there are still no other answers except the abolition of smoking, eating the right food and leading a good life.

\section{University of Edinburgh Gastrointestinal Unit}

However, it must not be supposed that the purpose of the physician in the management teams is to improve the performance of the surgeons. Collaboration works both ways with the surgeons bringing in their special skills, at the right time, to the benefit of the patient. Though for reasons previously stated it is not yet possible to show that the combined approach is an important step forward for all diseases, for certain diseases, particularly Crohn's disease, idiopathic ulcerative colitis, other small bowel diseases

TABLE 5. Percentage mortality in the treatment of bleeding peptic ulcer in two eras (after Vellacott et al., 1973)

\begin{tabular}{lcccc}
\hline & \multicolumn{2}{c}{$1975-77$} & \multicolumn{2}{c}{$1978-80$} \\
Site of ulcer & Conservative & Operated & Conservative & Operated \\
\hline Gastric & $7 \%$ & $28 \%$ & $7 \cdot 3 \%$ & $33 \%$ \\
Duodenal & $6.2 \%$ & $15.9 \%$ & $8 \cdot 1 \%$ & $15 \cdot 8 \%$ \\
\hline
\end{tabular}


with serious nutritional problems such as nonspecific ulcerative jejuno-ileitis and for chronic pancreatitis, the physician undoubtedly has a major role. There are surgeons who approach patient problems with a physician's regard for the importance of a detailed history and understand nutritional problems better than many physicians. The latter, however, have the time to feel their way into complex cases which the surgeon has to spend in the operating theatre. Both disciplines must be represented in the hospital nutrition team and indeed not only physicians and surgeons but also nurses, pharmacists and dietitians, as is now the case in most hospitals which have one.

Collaboration between doctors implies not just working together but bringing individual skills and different approaches to the resolution of case problems. Inevitably it educates and influences the whole team. It needs to be so arranged, however, that patients are protected from indecision and even disagreement. For these reasons it is important to consider the opinions of those who have worked in the Edinburgh Gastrointestinal Unit at the Western General Hospital as this was the first unit established for the specific purpose of management of all gastrointestinal disorders within a formal medical and surgical framework.

In 1949 arising out of their common interest in problems of gastroenterology, Wilfrid Card (physician and then Reader in the University of Edinburgh) and John Bruce (surgeon and then Senior Lecturer) conceived the idea of intimately combining the separate skills of physicians and surgeons at all stages in the management of patients with gastrointestinal disease. The Board of Management permitted the establishment of a combined unit, presently with 32 beds in two contiguous wards, one for each sex and both admitting surgical and medical patients. The wards were far from ideal and there was neither side-room accommodation nor laboratory facility. The principal objective was the 'creation of a specialized environment in which would be achieved a high standard of clinical care and in which no bed or patient is to be regarded as purely medical or purely surgical since all patients with gastrointestinal disease present aspects of significance for both physicians and surgeons'. It was however recognized that at different times in the natural history of disease in a particular patient one discipline could have a predominant role.

The physicians were to be responsible for most investigative procedures and the care of disorders which had no immediate surgical component, while decisions respecting operations were made after joint discussion. The responsibility for the final nature (and timing) of operations lay with the surgeons who also had charge of the post-operative care. Special problems, especially involving important pre-operative preparation and post-operative management were managed jointly throughout. The surgical and medical clinics were separate but there was a single waiting list for admission to the wards.

The teaching of undergraduates, postgraduates and general practitioners was also conducted jointly. $\frac{\bar{\omega}}{\partial}$ At a time when clinical skills in diagnosis and management were considerably more contributive than the results of investigations in the laboratory the joint approach in presenting patients, especially in postgraduate teaching exercises, was highly successful.

It was recognized by the founders that the unit was to a degree experimental and in a re-appraisal written eight years after its establishment W. I. Card and John Bruce (1958) wrote that 'neither of us would willingly return to the arrangement of separate wards'.

In 1954 on the appointment of a second physician $N$ two charges were created, the two seniors, W. I. Card 0 and $J$. Bruce forming one and the, then, assistant consultants the other (W. Sircus and C. W. A. Z Falconer).

The teams combined for the joint grand round of the week which in the early days involved perambulation from bed to bed, each patient problem being presented and discussed, and permitting the list $\vec{\phi}$ elective operations for the following week to b. drawn up.

To avoid the management of patients 'by committee', and to confirm for the individual patient that one person had overall responsibility for his welfare, each on admission was allocated to the charge of one of the physicians whether the context of the case problem was predominantly medical or surgical. If the patient had already been seen in the clinic of a particular physician or surgeon then on admission the patient continued in that person's care. Although the two teams were identified by the physicians, the surgeons came to have a somewhat more elastic commission, operations commonly being directed to one or other according to their special skills or interest.

In due course the growth of its clinical activity required a staff of three surgeons and three physicians. Whereas all the physicians have been full-time and practised only gastroenterology, the surgeons also provided the service for general surgical cases in $\mathcal{N}$ other wards and clinics with conventional arrange- $N$ ments and practice. Subsequently the Wolfson Foun- N dation provided extensive research laboratories and in 1969 the unit was officially named as the University of Edinburgh Gastro-intestinal Unit.

In the circumstances at the outset the 'special' investigative facilities consisted of one staff nurse who was trained in endoscopy practice and in 
methods of testing gastric and pancreatic function. All three were performed in a small room close by the wards. As a result of the growth of the unit over the next two decades and the technological progress of the speciality, the facilities presently include a purpose-built endoscopy suite staffed by three fulltime staff nurses and three auxiliaries and a purposebuilt clinical investigation suite with a sister and two staff nurses providing a wide range of diagnostic procedures. Furthermore, as the research work in the unit's laboratories has been closely related to the clinical problems of the service it has also provided certain diagnostic facilities: these have included oesophageal and colonic motility, gastrin immunoassay, breath hydrogen analysis and faecal fat analysis to name just a few. The laboratories have biochemistry and other technicians of various grades and in them research has been pursued by the training grades of surgeons as well as physicians, the work often forming the submission for ChM as well as $\mathrm{PhD}$ or MD.

When the laboratories were acquired the Regional Hospital Board agreed the appointment of a clinical scientist to administer them and to facilitate research (M. A. Eastwood) and a rich harvest of studies of bile metabolism and the role of fibre in health and disease has accrued. A large section of the laboratories is now devoted to immunological studies under the direction of another of the physicians (Anne Ferguson) and another to the investigation of the mechanisms underlying motility of the alimentary tract in health and disease, under the supervision of one of the surgeons (A. N. Smith).

Clinical research in the unit is facilitated by a records room staffed by part-time clerks, in which records of all patients whose disorders are the subject of ongoing clinical data research are identified and filed. Currently, for example, these include all cases of coeliac disease, inflammatory bowel disease, duodenitis and gastric ulcer but the subjects change as research programmes are completed and new ones are undertaken. A separate research office under the direction of one of the surgeons (W. P. Small) has the progress records of every patient submitted to surgery of the upper alimentary tract and provides surveillance information extending over 30 years.

Throughout the 34 years of the unit's existence the emphasis of the research has changed as new areas of interest arise and new expertise was imported into the unit in the change of staff. The research projects presently being pursued both within the laboratories and otherwise are listed to illustrate the surgical and medical spectrum which arises in a totally collaborated system (Table 6).

A feature over the last 15 years has been the arriving at all policy decisions of whatever nature,

TABLE 6. Current research projects in a combined medical-surgical gastrointestinal unit

Metabolism in the colon of wheat bran, gums and polysaccharides

The influence of the caecum on faecal output and constituents

The development of in vitro methods for measuring water phases in fruit and vegetable fibre

The measurement of enzymes in colonic mucosa

The effect of gum arabic metabolites on rat nutrition

Breath hydrogen and methane in neonates, breast fed and otherwise

Physiological evaluation of sphincter-saving excisions of the rectum

Manometric and electromyographic evaluation of paraplegic, geriatric, and post-radiotherapy disorders of recto-colonic function

Evaluation of monoclonal CEA antibody in detection of metastases in colonic cancer

Pulmonary function abnormalities in coeliac and inflammatory bowel disease

Vitamin E metabolism in coeliac disease

Intestinal permeability in sarcoidosis

The effect of exercise of patients on total parenteral nutrition

Change in faecal bile acids before and after cholecystectomy

Retrospective study of radiation enterocolitis (71 cases)

Trial of acetylcysteine for the treatment by mucolysis of chronic pancreatitis and cystic fibrosis

Overview of the results of a selective policy of procedure in peptic ulcer surgery

Review of highly selective vagotomy in ulcer management

Review of results of surgery of carcinoma of the colon

A comparative and combined study of gastric cancer in South-East Scotland and in Portugal

The relationship of gastroduodenal disease to dentition

A clinical and pathological review of 650 cases of idiopathic colitis and 350 cases of Crohn's disease managed over three decades

Morphological, clinical and physiological features of erosive duodenitis

The effect of omeprazole upon gastro-duodenal ulceration resistant to cimetidine and ranitidine

The clinical significance of gastric acid hypersecretion

Computer analysis of endoscopy practice including biliary and pancreatic procedures as well as upper and lower pan-endoscopy, both

diagnostic and therapeutic

The influence of $\mathrm{H}_{2}$ receptor antagonists on the long-term natural history of benign gastric ulcer

Induction and suppression of specific cell-mediated immunity by the feeding of antigen

Food allergic reactions and immunopathological mechanisms in atopic eczema and coeliac disease

Pathogenesis of the diarrhoea and malabsorption in giardiasis 
administrative or clinical, by discussion within an executive consisting of the six consultants, meeting over lunch for one hour each week. Once in each month the registrars, lecturers and senior nurses join the executive. The chairmanship of the executive is held for one year by rotation through the consultants. The consultant with administrative responsibility appointed by the Health Board attends to all the nonpolicy matters.

In order to eliminate as far as possible the personal prejudices or bias of the writer's, recorded confidential and prolonged interviews were conducted with the present and the surviving retired consultants of the unit, written questionnaires were completed by a number of other consultants whose gastroenterology training had been obtained in the Edinburgh unit, and by the present lecturers and senior registrars.

Despite these the evaluation was to an extent obscured by aspects which reflect the size of the staff of the unit as much as any other factor. The following commentary is a distillation of the mixture of opinions. There were many differences on details but the one in which there was absolute unanimity was that the setting up of a combined unit had been an important step forward and that in almost all specialist branches of medicine similar arrangements were required and should be established.

Six consultants with equal clinical status, each entitled to accompanied ward rounds, bound in different combinations within the two disciplines, can create difficulties in time, planning and communications for the junior doctors and the nursing staff. The number of consultants who can work together within a total collaboration team and achieve optimum efficiency is uncertain: much depends on personality and motivation. One combined gastrointestinal unit in the U.K. with the minimum possible staff of two consultants quickly came to grief when the approaches of the surgeon and the physician could not be reconciled: the plan had to be abandoned for several years but has recently started up again with a different surgeon and appears to be working well. Much also depends on the respective consultant's role: thus one consultant may be responsible for the greater part of the endoscopy load and required to do less ward work and clinic duties if a physician, and less operating theatre work if a surgeon. This is but an example. The impression is clear that where there are several surgeons and physicians it is inappropriate that each should have similar duties respecting endoscopy, clinic and ward duties, teaching and research.

The opinion is general among junior staff that 'the physicians stayed the surgeons' hands and the surgeons pushed the physicians into appropriate action'. The major advantage of this appears to be in the care of poor risk cases, in the guaranteed daily continuity of care through operating and nonoperating treatment phases, and in the management $c$. of complications such as in inflammatory bowel $\ddot{\vec{*}}$ disease with metabolic and nutritional problems. $\stackrel{\vec{F}}{\stackrel{9}{\rho}}$ Most senior and junior staff supported the present $\bar{C}$ non-selective admission policy, in which cholelithia- 흘 sis and uncomplicated peptic ulcer cases are placed $\frac{\bar{\omega}}{7}$ on the non-priority list for admission. Inevitably, $\stackrel{D}{\perp}$ however, because of the priority given to complex क management problems including those referred from $\rightarrow$ other hospitals, and the consequent waiting time for $?$ admission of uncomplicated problems, the general $\overrightarrow{\vec{\omega}}$ practitioners were conditioned to a policy of selective $\vec{\omega}$ referral. A few registrars, but no consultants, thought ${ }_{0}$ a policy of selective admissions would be preferable. 3

The surgeons believed cases were worked up better $\dot{\circ}$ than in a conventional unit, that there were far fewer explorative laparotomies than were made by the $\odot$ general surgical service, and that the seriously ill patient benefited by the ease of obtaining joint $N$ management review at frequent intervals in the day. 을 The ability to 'lean on one another' in difficult $\rightarrow$ clinical problems was clearly valued. By corollary it $z$ was thought that the collaborative system protected the patient against an individual doctor's imperfections.

Both senior and junior staff recognized the dangef of management decisions being delayed by 'commi tee' discussion between the surgeons and physiciaw⿳亠口冋. and the need to protect the patient from a commun cated sense of uncertainty. The early morning combined ward round of all patients by the surgical and medical registrars is important in this respect and also in identifying items to be communicated to the $\mathbb{D}$ consultants. During their respective ward rounds the $\stackrel{2}{\Rightarrow}$ consultants have to accept the need to record their opinions and management decisions in the common case-notes.

Young surgeons coming from a conventional surgical unit have found the jump enormous. 'In a 3 conventional unit when the surgeons decide $X$, then that is that and the matter is closed. In this unit when the surgeon says $X$ and the physician says $Y$, the matter is debated and a formula is reached,- -say $\frac{1}{2} Y+\frac{1}{2} X$.' The young surgeons find this approach $ᄋ$ disturbing, even confusing at first, especially when a definitive statement has to be made in the interest of $\frac{7}{2}$ the patient. There are casualties in the training process but the trainee surgeons become more aware of of a medical contribution to surgical matters and $N$ become less mechanistic in their own approach. The N registrars, on the other hand, thought that the wider $\omega$ spectrum of alimentary disorders resulted in more opportunities for research.

Many of those interviewed emphasized that an important feature of the combination of the disciplines includes to quote, the 'friction of the mind', a 
'stimulating intellectually-creative tension', though from time to time most of the consultants had thought, 'Oh for a quieter life'. Some registrars believed the public accounting that went on in the Grand Round protected patients from poor standards of care.

Almost all stressed that an important aspect of the system of total collaboration was the cross-fertilization with ideas. Competitive challenge between the consultants in the open forum of the Grand Rounds demanded a fair knowledge of the literature as well as a distillation of experience. The spin-off included the dissemination of information to all attending the Round, consultant and trainees alike. Thus most juniors stress that the benefits to the doctors are educational.

The opinion was general among the consultants that in such tight combinations the surgeons as well as the physicians needed to be full-time within the unit. The clinical involvement of the staff is heavy and to maintain a high academic standard more pairs of hands are required to allow the provision of blocks of time for research and writing.

It was generally recognized that the increasingly effective new methods of investigation have encouraged the maximum possible investigation of patient's problems before admission to the wards. Consequently a higher proportion of admissions have a stronger surgical content than in previous decades. Thus for the future the precise establishment of the consultants may also have to reflect such changes both in the respective members of each discipline required, and in the revision of their roles within the unit.

Throughout the three decades the unit has enjoyed an astonishingly selfless and expert service from the radiologists and pathologists in the hospital who also continuously educate the unit at each Grand Round.

Exercising of control by an executive committee of the consultants represents a democratic process and, like all such, leads to occasional difficulties in the making of decisions and weaknesses in the execution of them. Some considered it was possible that a head of department with an agreed authority might provide a better solution to leadership than a rotating chairman as at present. One young consultant had this to say, 'the big jerk is when you finally make a consultant appointment and you think, great! I will be my own boss at last, and then you find you have first to refer things to the executive or to the administration and that is resented'.

The consultants recognize that in return for the intellectual stimulation and the challenge there is a price to be paid for working in such close collaboration. It demands a considerable sacrifice of personal ease in reaching decisions respecting administrative matters, the promoting of research and the promotion of policy respecting matters of management.
Some overlap of duties is unavoidable so that extra work is involved. Above all, the system demands exclusion of the loner, the insecure and the fractitious and if such are imported into a system with total collaboration the effects can be severely disruptive.

It was an unanimous opinion that the right of the consultant in charge of a particular patient to investigate and treat that patient as he or she thinks is in their best interests, remains absolutely inviolable and without necessary regard to general policies of management that may have been agreed within the unit. Any problems from this source have been very rare indeed, perhaps because of the Grand Round, which, for two decades past, has been static and in which the 'public accounting' takes place.

That on the whole the difficulties are overcome is suggested not only by the survival of the unit and its essential internal arrangements after thirty-four years but by the continued referrals of patients from other consultants, including gastroenterologists, and from other hospitals elsewhere in the UK. Special investigative facilities account for some of these, for example, the facilities for manometric and electromyographic examination of ano-rectal function, but most seem to be a consequence of our combined experience.

Not a single member of the senior or junior staff believed that the combination had less than, or only as much to offer, as a conventional medical or surgical gastroenterology department.

Whatever the optimum size of staff should be or how the combination should be composed, neither of which was conceived unanimously, it was the unanimous belief that the patients gained considerable advantage from the arrangement and that, after all, must be the yardstick.

\section{Acknowledgment}

I would like to thank all those colleagues, senior and junior, whose opinions, time and trouble, were given so freely for the purpose of this paper. How the information has been used is solely my responsibility.

\section{References}

Birnie, C.G., Kennedy, F., Mackay, C. \& Watkinson, G. (1981) Experience of an upper gastro-intestinal bleeding management team. Gut, 22, 416.

CARD, W.I., BRUCE, J. (1958) An experiment in medical and surgical collaboration. American Journal of Digestive Diseases, 3, 528.

Himal, H.S., WATSON, W.W., Jones, C.W., MilleR, L. \& MACLEAN, L.D. (1974) The management of upper gastrointestinal haemorrhage. Annals of Surgery, 179, 489.

Hunt, P.S., Korman, M.G., Hansky, J., Marshall, R.D., Peck, G.G. \& MCCANN, W. (1979) Bleeding duodenal ulcer: reduction in mortality with a planned approach. British Journal of Surgery, 66, 663.

JONES, F. AVERY (1956) Haematemesis and melaena. Gastroentero$\log y, 30,166$. 
Jones, P.F., Johnston, S.J., McEwan, A.B., Kyle, J. \& NeedHAM, C.D. (1973) Further haemorrhage after admission to hospital for gastro-intestinal haemorrhage. British Medical Journal, 3, 660 .

MORRIS, D.L., HAWKer, P.C., DYKes, P.W. \& KeIGHLeY, M.R.B (1983) Upper gastro-intestinal bleeding-influence of a policy of centralisation. British Journal of Surgery, 70, 295.
TANNER, N.C. (1950) Gastro-duodenal haemorrhage as a surgical emergency. Proceedings of the Royal Society of Medicine, 43, 147.2 Vellacott, K.D., Dronfield, M.W., Atkinson, M. \& LangMAN, M.J.S. (1982) Comparison of surgical and medical manage ment of bleeding peptic ulcers. British Medical Journal, 284, 548. 